\title{
Representaciones Sociales Sobre Enseñanza Y Aprendizaje Que Sostienen Docentes De Nivel Medio Superior Y Superior
}

\author{
Juan Alberto Acosta Hernández (PhD) \\ Miriam Martínez Vázquez (MA) \\ Arturo Curiel Anaya (MA) \\ Aarón Reyes Rodríguez (PhD) \\ Anna Tarasenko (PhD) \\ Carlos Rondero Guerrero (PhD) \\ Universidad Autónoma del Estado de Hidalgo, México
}

Doi:10.19044/esj.2018.v14n25p1 ～URL:http://dx.doi.org/10.19044/esj.2018.v14n25p1

\begin{abstract}
This paper focuses on analyzing the social representations (SR) on mathematics, teaching and learning that have upper and middle level teachers who participated in a workshop on the use of a MOOC (Massive Open Online Course). It is used as an element of support for a Pre-Calculus face-to-face course. Using a questionnaire, the SR on the teaching and learning of mathematics were identified, as well as specific SR about the concept of inequality and the use of technology in the classroom. Based on the responses of the questionnaire, dimensions were captured that focuses on a particular meaning. This, therefore, is with the intention of organizing categories that would allow the establishment of hierarchies of the contents immersed in the field of SR of teachers.
\end{abstract}

Keywords: Social representations, Teaching, Learning, MOOC

\section{Resumen}

En esta investigación se analizan las representaciones sociales (RS) sobre las matemáticas, la enseñanza y el aprendizaje que tienen los docentes de nivel medio superior y superior quienes participaron en un taller sobre el empleo de un MOOC (Massive Open Online Course), como elemento de apoyo para un curso presencial de Pre cálculo. Con base en un cuestionario, se identificaron las RS sobre enseñanza y aprendizaje de las matemáticas, así como RS específicas sobre el concepto de desigualdad y uso de la tecnología en el salón de clases. Con base en las respuestas del cuestionario, se capturaron 
dimensiones que concentran un significado particular, con la intención de organizar categorías que permitieran establecer jerarquías de los contenidos inmersos en el campo de las RS de los docentes.

Palabras clave: Representaciones sociales, Enseñanza, Aprendizaje, MOOC.

\section{Introducción}

Estudiar la relación entre la enseñanza y aprendizaje de las matemáticas se ha considerado como un campo de investigación relevante (Hiebert \& Wearne, 1993). De igual forma, resulta interesante profundizar en el estudio del aspecto social ligado a la enseñanza de esta ciencia, razón por la cual se llevó a cabo la presente investigación. A través de este estudio se pretende atender el estudio de la vida cotidiana y el conocimiento del sentido común asociados a la matemática escolar (Martínez, 2011) dentro de una comunidad de práctica, ya que este conocimiento y sus respectivas imágenes, creencias y representaciones, son indicadores de las formas de pensar de los profesores y actúan como guía del actuar docente en el salón de clases (Piña \& Cuevas, 2004). Como expresa Mireles (2015), "el conocimiento de sentido común, que se usa en la vida cotidiana, es un objeto de indagación relevante porque permite conocer la realidad social desde el punto de vista de los actores y sus vínculos con las circunstancias sociales."

Comprender la forma en que profesores de bachillerato y universidad, para quienes no existen programas específicos de formación en México, conceptualizan el aprendizaje y enseñanza de las matemáticas desde la perspectiva del conocimiento de sentido común es importante, ya que las RS influyen en la manera de reconocer los actos ligados al aprendizaje de los profesores, como integrantes de una comunidad de práctica, que comparten algún interés, un conjunto de problemas, o la pasión por un tópico, y quienes profundizan su conocimiento y experiencias interactuando de forma continua (Wenger, McDermott \& Snyder, 2002).

El pensamiento natural o sentido común asociados a la matemática escolar, en ocasiones, es contrario al pensamiento científico, ya que se constituye desde las experiencias de los sujetos, de las informaciones, conocimientos, y modelos de pensamiento que se reciben y transmiten desde la tradición, la propia experiencia educativa y la comunicación social. Así, este conocimiento es, en muchos aspectos, socialmente elaborado y compartido. En cierto sentido, se trata de un conocimiento práctico (Jodelet, 1986). Recuperar las RS de las personas y resignificarlas, más allá de las normas academicistas tradicionales, es relevante para el esclarecimiento de la construcción del conocimiento matemático en un ámbito social, ya que estudiantes y profesores, de cualquier nivel educativo, se acercan al conocimiento matemático a partir 
de un conjunto de ideas, creencias, conocimientos y conjeturas basados, fundamentalmente, en un pensamiento natural.

Analizar las RS de los docentes es importante porque éstas determinan su actuación en el aula y porque dicho conocimiento no ha sido ampliamente revisado en el contexto mexicano. Además, profundizar en el tema de las RS que sostienen los profesores sobre la enseñanza y aprendizaje de las matemáticas, podría facilitar la comprensión de las dificultades de aprendizaje en la disciplina y podría ser el punto de partida para la transformación de la práctica en el aula.

Adoptamos la definición de RS propuesta por Jodelet (1986, p. 474), quien considera que una RS “designa una forma específica de conocimiento, el saber de sentido común, cuyos contenidos manifiestan la operación de procesos generativos y funcionales socialmente caracterizados". Una RS es una organización de imágenes y de lenguaje que simboliza y encapsula actos y situaciones que son y se convierten en comunes (Moscovici, 1979). Las representaciones sociales producen y determinan comportamientos, porque definen la naturaleza de los estímulos que nos rodean. "La representación social es una modalidad particular del conocimiento, cuya función es la elaboración de los comportamientos y la comunicación de los individuos" (ibid, p. 17).

\section{Antecedentes}

La investigación educativa recientemente se ha interesado por estudiar las RS en el contexto escolar. Particularmente, existen estudios que analizan las RS de estudiantes con respecto a su lugar en el aula de ciencias (Bender, Defago \& Cutrera, 2009); y las RS sobre la educación a distancia sostenidas por estudiantes de grado y posgrado (Bron, 2016). También hay investigaciones acerca de RS de alumnos que emplean herramientas en línea, creadas exprofeso, como apoyo a cursos presenciales de matemáticas introductorias de ingeniería (Acosta, Martínez, Tarasenko \& Curiel, 2017 ); trabajos sobre la relación entre las RS con respecto a la enseñanza y aprendizaje de la física y la química a estudiantes de secundaria, con la práctica docente de sus profesores (Guirado et al., 2013b); RS de la matemática sostenidas por estudiantes de ingeniería (Heredia \& Fernández, 2017); RS de estudiantes para profesor acerca de la enseñanza y aprendizaje de las ciencias (Mazzitelli, 2013). En el caso de investigaciones que analizan RS de estudiantes sobre la enseñanza y aprendizaje, Martínez (2011), identificó que los estudiantes de bachillerato perciben el aprendizaje de las matemáticas como algo ligado a la utilidad para resolver problemas de la vida cotidiana mientras que la enseñanza se representa en términos de la metáfora de la transferencia a través de la explicación, de un bien o una posesión por parte de quien enseña. 
En lo que respecta a las RS de docentes, los temas son variados: RS sobre la diversidad escolar (Apablaza, 2014); RS sobre reformas en el área de matemáticas (Ávila, 2011); RS de la lectura (Bacilio, 2015). Particularmente, en lo que respecta a la investigación relacionada con RS de profesores sobre la enseñanza y aprendizaje, existen investigaciones en áreas como las ciencias naturales (Aguilar et al., 2011); las ciencias sociales (Ramírez, 2016; Verdugo, 2016); física y química (Guirado et al., 2013a).

En la última década ha crecido el interés en hacer investigación sobre las RS en torno a la educación (Martínez, 2011; Garnique, 2012), donde la mayoría de ellas se han enfocado al ámbito general de educación y otras a la matemática educativa. Como expresa Moscovici (1979, p. 40), "El reto de la ciencia hoy día es ya no hacer más científico el conocimiento común, sino hacer más común lo científico”.

Las investigaciones que abordan las RS se han incrementado, aunque su repercusión en el ámbito educativo debería ser mayor de acuerdo a lo que requiere el entorno de una sociedad del conocimiento. En particular, los estudios que pretenden repercutir en los procesos del aprendizaje, sobretodo en el de la matemática, resultan de gran importancia.

\section{Marco Teórico Referencial}

La teoría de las RS considera que toda realidad es representada, esto es, apropiada por el individuo o el grupo, reconstruida en su sistema cognitivo e integrada en su sistema de valores. Las RS están constituidas por un conjunto de informaciones, creencias, opiniones y actitudes, conscientes o no, a propósito de un objeto o fenómeno determinado (Ávila, 2001). De igual manera, las RS establecen una forma muy particular de conocimiento humano, denominado "conocimiento del sentido común" (Jodelet, 1986, p. 473; Guimelli, 2004, p. 63). Se caracterizan por englobar creencias, conocimientos y opiniones producidas y compartidas por los individuos de un mismo grupo con respecto a un objeto social en particular (Guimelli, 2004).

Las RS son un cuerpo organizado de conocimientos y una de las actividades psíquicas gracias a la cual los seres humanos hacen inteligible la realidad física y social, se integran en un grupo social o en una relación cotidiana de intercambios (Moscovici, 1979); estas constituyen una preparación para la acción, en la medida que guían el comportamiento, remodelan y reconstituyen los elementos del medio en el que tal comportamiento tiene lugar. Las RS producen y determinan comportamientos ya que definen la naturaleza de los estímulos que nos rodean y el significado de las respuestas que debemos darles; son una modalidad particular de conocimiento, cuya función es la elaboración de los comportamientos y la comunicación entre los individuos (ibid, pp. 17, 32). 
Las RS, a diferencia de las representaciones colectivas de Durkheim, poseen un carácter dinámico (Moscovici, 1979). El carácter dinámico de las RS implica que las personas "construyen significados y teorías sobre la realidad en una relación dialéctica entre lo individual y lo social” (Aguilar et al., 2011, p. 2). Los estímulos que se reciben del medio se someten a un proceso de transformación, de evolución, para convertirse en conocimiento que se emplea durante la vida cotidiana. Durante el proceso de empleo, los conceptos se objetivan, conformando "la textura de lo que la realidad es para cada uno" (Moscovici, 1979, p. 33). El objetivo de las representaciones sociales no es hacer avanzar el conocimiento, sino "estar al corriente", no ser ignorante, al integrar conceptos en un cuadro coherente de lo real o contar con un lenguaje que permita hablar de lo que habla todo el mundo dentro de una comunidad de práctica, haciendo que el mundo sea lo que pensamos que es o lo que debe ser (Moscovici, 1979, p. 39).

Una de las características de las $\mathrm{RS}$ es la producción de comportamientos y relaciones con el medio. Esta es una acción que modifica a ambos y no una reproducción de estos comportamientos o de estas relaciones, ni una reacción a un estímulo exterior dado. Una RS es siempre una representación de alguien, así como es la representación de una cosa, lo cual quiere decir que la estructura cognitiva de las personas incidirá en el contenido y organización de los elementos constituyentes de la RS. De este modo, las RS hacen posible que el sujeto que conoce se coloque dentro de aquello que conoce (Moscovici, 1979, pp. 17, 33, 43).

Las RS están compuestas por tres dimensiones: información, actitud y campo de la representación o imagen. La información, es la organización de los conocimientos que posee un grupo con respecto a un objeto social. La actitud es el contexto de opinión, que refiere la determinación a favor o en contra en cuanto al objeto de las RS. Esta es la componente más aparente, fáctica y conductual de la representación, y la cual suele estudiarse más, por su vertiente conductual y motivacional. La actitud es la más frecuente de las tres dimensiones y, quizá, primera desde el punto de vista genético, lo cual quiere decir que se representa una cosa únicamente después de haber tomado posición $\mathrm{y}$ en función de la posición tomada (Moscovici, 1979, p. 49). El campo de representación se refiere al contenido concreto y limitado de las proposiciones sobre un aspecto preciso del objeto de la representación. Muestra el establecimiento de la composición de la representación de forma estratificada, variando de grupo a grupo. Concede también vislumbrar las características del campo que constituye la información, esto es: la idea de imagen, de modelo social, contenido concreto y limitado de las propuestas en las que se hace referencia precisa del objeto de representación. También en este apartado se pueden considerar los aspectos ideológicos en el encuadramiento del campo de representación. 
Para la formación de las RS cada persona parte de las observaciones y, sobre todo, de los testimonios que se acumulan a propósito de acontecimientos corrientes. Una representación social se elabora de acuerdo con dos procesos fundamentales: la objetivación y el anclaje (Moscovici, 1979, p. 75). La objetivación lleva a hacer real un esquema conceptual, a duplicar una imagen con una contrapartida material. Objetivar es reabsorber un exceso de significaciones materializándolas. También es trasplantar al plano de la observación lo que era sólo inferencia o símbolo. Naturalizar y clasificar son dos operaciones esenciales de la objetivación. Una convierte en real al símbolo y la otra da a la realidad un aspecto simbólico. Una enriquece la gama de imágenes asociadas al objeto representado, mientras que la otra separa los atributos del objeto para poder conservarlos en un cuadro general de acuerdo con un sistema de referencias instituido socialmente (Moscovici, 1979, p. 77). El anclaje designa una ciencia en una jerarquía de valores y entre las operaciones realizadas por la sociedad. En otros términos, a través del proceso de anclaje, la sociedad cambia el objeto social por un instrumento del que puede disponer, y este objeto se coloca en una escala de preferencia en las relaciones sociales existentes. El proceso de anclaje transforma el objeto de representación en un saber útil para todos. En otras palabras, la objetivación traslada el objeto de representación al dominio del ser, mientras que el anclaje la traslada al dominio del hacer (Moscovici, 1979, p. 121).

La noción de RS ubica el momento en que se intersectan las vertientes social con la psicológica. Esto es, lo concerniente a la manera en que los humanos aprenden los hechos cotidianos, las características del escenario, los datos que en él influyen y los individuos en el sistema. Dicho de otro modo, el saber "ingenuo", "espontáneo", que es de mucho interés para las ciencias sociales, llamado pensamiento natural o sentido común, es opuesto al pensamiento científico. Este saber, además de establecerse desde las experiencias, tanto individuales, como colectivas, también se constituye a partir de la tradición, la educación y la comunicación social. Por ello, este conocimiento es, desde diversos puntos de vista, un saber socialmente elaborado y compartido (Jodelet, 1986, p. 473).

El intentar recobrar las creencias de las personas y reconocer los sustentos teóricos que las soportan, desde el aspecto de los estándares de la cientificidad, es un beneficio para las ciencias sociales; y en particular para la educación matemática, lo es hacer más común los saberes científicos. En muchas ocasiones, en el ambiente del aula, el sentido común predomina sobre el intelecto y sobre la construcción de un conocimiento científico. En virtud de tal, la conducción de los aprendizajes no siempre guía al profesor universitario, ya que como ser social, se efectúan acciones que están basadas en las experiencias escolares que ha tenido con anterioridad. Dicho de otra manera, es la noción de sentido común originada en el intercambio de expresiones del 
grupo social que pretende comunicar, estar al día y sentirse ubicado en el ámbito social. Es una forma de saber por medio del cual el sujeto se ubica dentro de lo que él conoce; tiene además dos aspectos, uno figurativo y otra simbólico, lo cual posibilita acreditar a toda figura un sentido y a un sentido una figura (Rodríguez, 2001).

Desde el punto de vista esquemático, las RS surgen cuando los individuos discuten acerca de temas de interés común o cuando hay la percepción de que los hechos elegidos son significativos por quienes controlan el ámbito de comunicación (Farr, 1993, en Mora 2002). De igual manera, las RS no son simples opiniones o actitudes; estas forman parte de un sistema lógico cognitivo con lenguaje propio, y son ramas del conocimiento con propósitos de descubrir y organizar la realidad. Constituyen sistemas de valores, ideas y prácticas con doble funcionalidad: la primera, el establecimiento de un orden que posibilite al individuo orientarse en su mundo material y social y tener injerencia en él; y la segunda, la factibilidad de transmitir entre miembros de grupos sociales una norma para denominar y catalogar sin equívoco los diferentes aspectos de su mundo y su cronología, tanto individual como grupal.

\section{Metodología}

La recolección de los datos se llevó a cabo a través de un cuestionario, el cual se integró por diez preguntas. Este cuestionario se aplicó en el contexto de una comunidad de práctica, constituida por docentes de matemáticas, de bachillerato y licenciatura, quienes acudieron a un taller de cuatro horas sobre "Experiencias en el diseño de contenidos de un MOOC de Pre cálculo", organizado por una universidad pública, durante el mes de diciembre del año 2017. Este taller tiene su origen en los altos índices de reprobación de la asignatura de Pre cálculo, la cual se integró al currículo de cinco licenciaturas, en la universidad objetivo, desde el año 2013. La opinión de los docentes que integran la Academia de Matemáticas es que realmente los estudiantes no tuvieron una ganancia real en conocimientos y sus actitudes de responsabilidad no mejoraron (Rondero, et al., 2015, pp. 96-99). Por otro lado, recopilando las críticas de los mismos actores, se concluye que, durante el proceso de preparación para la implementación del nuevo modelo, no hubo una capacitación disciplinar docente adecuada que permitiera abordar la implementación.

Debido a la problemática de reprobación, la Academia de Matemáticas Básicas y el Grupo de Investigación en Tecnologías Avanzadas Aplicadas a la Educación de la universidad decidieron construir un MOOC (Massive Open Online Course) de Pre cálculo (Acosta, Curiel, Martínez, Pozas, \& Tarasenko, 2018), como un instrumento que permitiera colaborar en la solución de algunas de las dificultades de aprendizaje de los estudiantes de la institución, y cuya 
utilización pudiera, extenderse a otros programas educativos o a estudiantes de otras instituciones; esto hasta llegar a ser un curso virtual autónomo en cuanto a los contenidos, prácticas educativas, ejercicios, evaluaciones y autoevaluaciones. La función del MOOC es fortalecer, en un medio virtual, las vertientes del triángulo didáctico: alumno, maestro y saber (Chevallard, 2000); los temas fueron seleccionados minuciosamente de manera previa, como tópicos muestra.

Durante el taller se llevó a cabo un proceso de discusión que giró en torno a la enseñanza y aprendizaje de las desigualdades, y la forma en cómo un MOOC, diseñado por investigadores de la universidad, podría apoyar el entendimiento de los estudiantes acerca del tema (Acosta et al., 2018). La toma de datos se llevó a cabo al finalizar el taller, porque se consideró que el conocimiento de sentido común - en particular las RS - se elabora en vinculación indisociable con un entorno; por lo tanto, determinar ese contexto es uno de los primeros quehaceres del investigador (Mireles, 2015). En el taller participaron 29 docentes, pero solo 13 de ellos accedieron a responder el cuestionario. Siete de los docentes trabajan en nivel bachillerato, cinco en nivel licenciatura y uno en ambos niveles. El grado máximo de estudios de los profesores fue licenciatura en cinco casos, maestría en siete y doctorado en uno.

Las preguntas se diseñaron de forma que generaran discursos escritos que permitieran conocer las RS que se establecen desde el lenguaje escrito como reflejo de la vida cotidiana y del sentido común de los docentes de matemáticas, ya que como argumenta Martínez (2011, p. 96): "El discurso, pues, es portador privilegiado de las representaciones sociales que circulan en el universo simbólico de los estudiantes.". Al analizar las RS se intenta conocer cómo piensan los sujetos sobre un determinado objeto de representación, y esto se reconoce como un acto de construcción, no como un reflejo, ni como producto de un determinismo social (Mireles, 2015).

Las preguntas del cuestionario fueron las siguientes:

1) ¿Qué es aprender matemáticas?

2) ¿Qué es enseñar matemáticas?

3) ¿Por qué aprender matemáticas?

4) ¿Cómo se aprende a resolver desigualdades?

5) ¿Cuáles son los tipos de desigualdades que más se les complican a los estudiantes y por qué?

6) ¿Consideras que la herramienta del MOOC ayuda con el aprendizaje del tema de desigualdades?

7) ¿Cuántas veces crees que se deba trabajar con el MOOC o con el profesor de forma presencial?

8) ¿Consideras que los estudiantes preferirían estudiar el tema con el MOOC o con el profesor de forma presencial? 
9) ¿Los instrumentos de evaluación del MOOC fueron suficientes y claros?

10) ¿Cuáles son tus sugerencias para mejorar el MOOC?

Se considera que con estas preguntas es posible identificar e interpretar la comprensión cotidiana o sentido común del conocimiento, respecto a la enseñanza y el aprendizaje, que sostienen los docentes que asistieron al taller. A pesar de que la relación, estructura y orden que guardan los conocimientos, constituyen su aspecto formal; es decir que todas las ciencias tienen una forma, una columna vertebral que las sostiene; esa estructura está dada por la razón. Pero el contenido de las ciencias factuales son los hechos y a ellos se tiene acceso mediante la experiencia. Las preguntas del cuestionario son abiertas, con el propósito de no acotar las respuestas de los participantes, y dejarlos que se comuniquen con total libertad; pero están contextualizadas en alguna de las dos vertientes del contexto de opinión.

Se sugirieron tres preguntas generales con el propósito de saber la RS de aprendizaje de las matemáticas (1, 2 y 3), dos preguntas para conocer la RS sobre las dificultades de los estudiantes con el tema de desigualdades $(4,5)$ y tres preguntas para determinar cómo el MOOC podría ayudar a superar esas dificultades $(6,7,10)$. Se sugirió una pregunta para conocer la preferencia entre usar el MOOC o implementar una sesión presencial (8), y finalmente una pregunta respecto de la evaluación de los aprendizajes (9).

La investigación fue de corte cualitativo; los docentes visualizaron el MOOC de Pre cálculo y pudieron interactuar con él durante una sesión de dos horas. Posteriormente, se aplicó el cuestionario de manera escrita. De acuerdo con Álvarez-Gayou (2009), la opción más adecuada para este tipo de investigación es mediante cuestionarios con preguntas abiertas.

\section{Resultados}

La pregunta ¿Qué es aprender matemáticas?, produjo los siguientes comentarios generalizados que se convirtieron en categorías: El 21\% de los docentes considera que es conocer herramientas para resolver problemas en diversos entornos, para el 50\% es razonar, crear, resolver, desarrollar, modelar situaciones de la vida cotidiana, para el $21 \%$ también es comprender los números reales, realizar operaciones para conocer el universo y sólo para el $7.1 \%$ es dar solución a una desigualdad.

En la segunda pregunta ¿Qué es enseñar matemáticas? el 57\% de los docentes piensa que es posibilitar la adquisición de conocimiento matemático, mediante herramientas y métodos para resolver problemas, mientras que en un porcentaje de $14 \%$, sostienen que es construir actividades para que los estudiantes identifiquen patrones, relaciones y emitan conjeturas y por otro lado es motivar a los estudiantes, despertar su interés por conocer, comprender y aplicar los objetos matemáticos; sólo un docente afirma que es dar leyes, 
hipótesis, axiomas y otro más considera que es guiar al estudiante a ver el mundo desde otra perspectiva.

Para la tercera pregunta: ¿Por qué aprender matemáticas?, el 31\% de los docentes explica que las situaciones cotidianas y profesionales involucran siempre el uso de herramientas matemáticas, el 19\% considera que para resolver problemas, mientras que el $25 \%$ supone que son necesarias para comprender el entorno mejorarlo y, con el mismo porcentaje, otro grupo determina que las matemáticas ayudan a desarrollar habilidades como el razonamiento y la generalización, pero también conocimiento.

La cuarta pregunta: ¿Cómo se aprende a resolver desigualdades?, el $25 \%$ sostiene que comparando y aplicando las reglas de desigualdades, por otro lado el 56\% afirma que con la explicación conceptual, algebraica, gráfica y sus aplicaciones y el 19\% resolviendo ejercicios.

La quinta pregunta, ¿Cuáles son los tipos de desigualdades, que más se les complican a los estudiantes y por qué?, un docente respondió que los problemas de intersección, para otros son las fraccionarias con números negativos, con dos variables, sin solución o con solución infinita o todas, para dos docentes son las dobles y para otros dos son las de valor absoluto; tres docentes afirman que son las cuadráticas y otros tres las racionales.

En la sexta pregunta: ¿Consideras que la herramienta del MOOC ayuda con el aprendizaje del tema de desigualdades?, todos los docentes consideran que sí y algunos expresan opiniones como que el MOOC tiene una accesibilidad permanente y refuerza el aprendizaje.

La séptima pregunta: ¿Cuántas veces crees que se deba trabajar con el MOOC para atender el tema de desigualdades?, el 33\% propone que un par de veces, mientras que el $6.7 \%$ opina que tres, el $27 \%$ que las veces que sean necesarias y el $33 \%$ que depende del estudiante.

La octava pregunta: ¿Consideras qué los estudiantes preferirían estudiar el tema en el MOOC o con el profesor de forma presencial?, el $47 \%$ establece que con el profesor, el 29\% considera que el MOOC es una actividad para reforzar lo visto de forma presencial y el $24 \%$ indica que ambos son necesarios.

La novena pregunta: ¿Los instrumentos de evaluación del MOOC fueron suficientes y claros?, el 73 \% comenta que sí; por otro lado el 18\% afirma que no, pues es necesario aumentar y diversificar los instrumentos y el $9.1 \%$ determina que depende del tema.

En la última pregunta: ¿Cuáles son tus sugerencias para mejorar el $M O O C$ ?, las opiniones fueron diversas; un profesor pide un MOOC enfocado para preparatoria, otro sugiere más ejercicios en cada tema, otro más propone agregar un apartado de sugerencias y/o comentarios y uno más pide incluir videos de los temas con estudiantes, mientras que el $17 \%$ solicita que se escriban introducciones, reglas y ejemplos; el $8.7 \%$ demanda que se empleen 
colores, el $13 \%$ requiere que se mejore la calidad de audio y video y finalmente el 35\% quiere que sean más explícitos los procedimientos y explicaciones.

\section{Conclusion}

En este trabajo de investigación se han presentado algunos resultados acerca de las RS, como "contextos de opinión” con propósitos didácticos, al momento de hacer uso de un MOOC de Pre cálculo, a partir de una muestra no estadística de docentes que participaron en el taller: "Experiencias en el diseño de contenidos de un MOOC de Pre cálculo". El MOOC se ha venido empleando desde el año 2013 como alternativa para coadyuvar a resolver la problemática del aprendizaje del respectivo curso presencial en la Universidad Autónoma del Estado de Hidalgo (UAEH). El cuestionario se diseñó con preguntas abiertas, ya que se pretendió no acotar las respuestas de los participantes, y dejarlos que se comunicaran con absoluta libertad. Las preguntas están contextualizadas en alguna de las dos vertientes del contexto de opinión. Los profesores que respondieron el cuestionario demuestran una actitud de asociar el aprendizaje de la matemática con razonar, crear, resolver, desarrollar, modelar situaciones de la vida cotidiana. En la segunda pregunta, en cuanto a qué es enseñar matemática, la mayoría de los docentes piensa, que es posibilitar la adquisición de conocimiento matemático, mediante herramientas y métodos, para resolver problemas, en tanto que en menor porcentaje, sostienen que es construir actividades para que los estudiantes identifiquen patrones, relaciones y emitan conjeturas; y además motiven a los estudiantes a despertar su interés por conocer y comprender y que apliquen los objetos matemáticos. Respecto a por qué aprender matemáticas, los docentes piensan que las situaciones cotidianas y profesionales involucran siempre el uso de herramientas matemáticas y suponen que son necesarias para comprender el entorno y mejorarlo, además consideran que permiten resolver problemas y que las matemáticas ayudan a desarrollar habilidades como el razonamiento y la generalización, pero también conocimiento. La cuarta pregunta, que se refiere a cómo se aprende a resolver desigualdades, la mayoría de encuestados sostiene que con la explicación conceptual, algebraica, gráfica y sus aplicaciones y en menor proporción afirmaron que resolviendo ejercicios. En la pregunta¿cuáles cree que son los tipos de desigualdades, que más se les complican a los estudiantes y por qué?, las respuestas fueron diversas, no habiendo algún tipo totalmente predominante. La respuesta a la sexta pregunta, está unificada en el sentido de que todos los docentes coinciden en que el MOOC ayuda pues tiene una accesibilidad permanente y refuerza el aprendizaje. En lo que respecta a las veces que se debe trabajar con el MOOC para atender el tema de desigualdades, las respuestas de mayor predominio son que ello depende del estudiante o un par de veces. Respecto a la octava pregunta, coincide con los resultados de un estudio similar (Acosta, et al, 
2017), en el sentido, de preferir trabajar "con el profesor" y que consideran que el MOOC es una actividad para reforzar los contenidos revisados de forma presencial; también consideran que ambos son necesarios. Respecto a la novena dimensión, casi tres cuartas partes de los docentes afirman que los instrumentos de evaluación del MOOC son claros. El comentario más común respecto de la última pregunta es que se deben explicitar los procedimientos y explicaciones del MOOC.

El aprender a resolver desigualdades elementales se vincula con el esfuerzo y la solución de ejercicios y problemas, y con el empleo y relación entre representaciones semióticas, ya que la construcción de conexiones es un indicador de entendimiento. En sus respuestas resaltan la característica esperada de que quien enseña, debe hacer comprensible los temas desarrollados. Esta es la gran demanda de la didáctica, que se nutre de los trabajos en la línea de las RS, aunque su incidencia en el ámbito de la educación matemática debería ser mucho mayor de acuerdo a lo que está demandando nuestro entorno social. Como ya sedijo: El reto de la ciencia hoy día es ya no hacer más científico el conocimiento común, sino hacer más común lo científico (Moscovici, 1979), y esto cae muy bien en el plano didáctico.

\section{References:}

1. Acosta, J., Curiel, A., Martínez, M., Pozas, M., \& Tarasenko, A. (2018). Un MOOC de Pre cálculo. (Número de registro de INDAUTOR: 03-2018-032312493700-01). Recuperado el 13 de junio de 2018 de http://cidecame.uaeh.edu.mx/cursos/precalculo/

2. Acosta, J.A., Martínez, M., Tarasenko, A., \& Curiel, A. (2017). Las Representaciones Sociales mostradas a través de un MOOC de Pre cálculo. Revista Novuscientífica 6.0, 4(1), p. 34-58.

3. Aguilar, S. B., Mazzitelli, C. A., Chacoma, M. S., \& Aparicio, M. T. (2011). Saberes del docente y representaciones sociales: implicancias para la enseñanza de las ciencias naturales. Revista Electrónica “Actualidades Investigativas en Educación”, 11(2), 1-28.

4. Alvárez-Gayou, J. L. (2009). Cómo hacer investigación cualitativa: fundamentos y metodología (Reimpresión). México: Paidós.

5. Apablaza, M. (2014). Representaciones sociales de profesores respecto a la diversidad escolar en relación con los contextos de desempeño profesional, prácticas y formación inicial. Estudios Pedagógicos, 40(1), 7-24.

6. Ávila, A. (2001). Los profesores y sus representaciones sobre la reforma a las matemáticas. Perfiles Educativos, 23(93), 59-86.

7. Bacilio, J.C. (2015). Representaciones sociales de la lectura en docentes de nivel primaria. RIDE Revista Iberoamericana para la 
Investigación y Desarrollo Educativo, 6(11). Recuperado el 8 de junio de 2017 de http://www.redalyc.org/articulo.oa?id=498150319014

8. Bender, G., Defago, A., \& Cutrera, G. (2009). Representaciones sociales en el aula de ciencias. El lugar del alumno. Enseñanza de las Ciencias, Número Extra VIII Congreso Internacional sobre Investigación en Didáctica de las Ciencias, 996-1000.

9. Bron, M. (2016). Representaciones sobre la educación a distancia: El caso de la Universidad Nacional de la Rioja (Argentina). Revista Linhas. Florianópolis, 17(33), 48-81.

10. Chevallard, Y. (2000). La Transposición Didáctica: del saber sabio al saber enseñado. Buenos Aires: Editorial AIQUÉ.

11. Durkheim, É. (2001). Las reglas del método sociológico. México: Fondo de Cultura Económica.

12. Farr, R.M. (1993). Theory and method in the study of social representations. In G.M. Breakwell \& D.V. Canter (Eds.), Empirical Approaches to Social Representations (pp.15-38). Oxford: Clarendon Press.

13. Garnique, C. (2012). Las representaciones sociales. Los docentes de educación básica frente a la inclusión escolar. Perfiles Educativos, 34 (137), 99-118.

14. Guimelli, C. (2004). El pensamiento social (1a ed.) [Filosofía y Cultura Contemporánea]. México: Ediciones Coyoacán.

15. Guirado, A. M., Mazzitelli, C. A., \& Olivera, A. C. (2013). Representaciones sociales y práctica docente: una experiencia con profesores de física y química. Revista de Orientación Educacional, 27(51), 87-105.

16. Guirado, A. M., Mazzitelli, C. A., Olivera, A. C., \& Quiroga, D. P. (2013). Relaciones entre las representaciones de los alumnos acerca de la enseñanza y aprendizaje de la física y de la química y la práctica docente. Revista Electrónica de Enseñanza de las Ciencias, 12(2), 347361.

17. Heredia, W. E. \& Fernández, P. (2017). Representación social de la matemática en estudiantes de ingeniería: un estudio exploratorio en cursos propedeúticos. Transformación, 13(1), 17-31.

18. Hiebert, J. \& Wearne, D. (1993). Instructional tasks, classroom discourse, and students' learning in second-grade arithmetic. American Educational Research Journal, 30(2), 393-425.

19. Jodelet, D. (1984-1986). La representación social: fenómenos, conceptos, y teoría. En S. Moscovici (Ed.) Psicología social II: Pensamiento y vida social. Barcelona: Paidós.

20. Moscovici, S. (1979). El psicoanálisis, su imagen y su público (Trad. Nilda María Finetti). Buenos Aires: Huemul. 
21. Martínez, G. (2011). Representaciones sociales que poseen estudiantes de nivel medio superior acerca del aprendizaje y enseñanza de las Matemáticas. Perfiles Educativos, 33(132), 90-109.

22. Mazzitelli, C. A. (2013). Representaciones acerca de la enseñanza y aprendizaje de las ciencias durante la formación docente inicial. Profesorado Revista de Currículum y Formación de Profesorado, 16(3). Recuperado el 8 de junio de 2018 de http://www.ugr.es/local/recfpro/rev163COL10.pdf

23. Mireles, O. (2015). Metodología de la investigación: operaciones para develar representaciones sociales. magis, Revista Internacional de Investigación en Educación, 8(16), 149-166.

24. Mora, M. (2002). La teoría de las representaciones sociales de Serge Moscovici. Athenea Digital, (2), 1-25. Recuperado el 11 de junio de 2018 de http://atheneadigital.net/article/view/n2-mora/55-pdf-es

25. Piña, J. M. \& Cuevas, Y. (2004). La teoría de las representaciones sociales. Su uso en la investigación educativa en México. Perfiles Educativos, 26 (106), 102-124.

26. Ramírez, M.I. (2016). Representaciones sociales acerca de la enseñanza de las ciencias sociales. Tesis de maestría, Universidad Autónoma de Manizales. Colombia.

27. Rodríguez, T. (2001). Las razones del matrimonio. Representaciones, relatos de vida y sociedad. México: Universidad de Guadalajara

28. Rondero, C., Reyes, A., \& Acosta, J.A. (2015) Seguimiento de una innovación curricular: una asignatura de matemáticas. European Scientific Journal, 11 (6), 95-115.

29. Verdugo, Y. F. (2016). Las representaciones sociales de las prácticas pedagógicas de los docentes de ciencias sociales en la media vocacional en el colegio de Nuestra Señora de Fátima Cartagena. Tesis de maestría no publicada. Bogotá: Universidad Santo Tomás.

30. Wenger, E., McDermott, R., \& Snyder, W. M. (2002). A guide to managing knowledge: Cultivating communities of practice. Boston, MA: Harvard Business School Press. 
Evaluación de la percepción social del empleo del MOOC en la parte de desigualdades DIMENSIONES

¿Qué es aprender matemáticas?

Conocer herramientas para resolver problemas en diversos entornos \begin{tabular}{lll|l|l|l}
1 & 1 & 1 & 1
\end{tabular} \begin{tabular}{|l|l|l|l|l|l|l|l|l|l|}
\hline & & & & & & & & & 3 \\
\hline
\end{tabular}

Razonar, crear, resolver, desarrollar, modelar situaciones de la vida cotidiana

Comprender los números reales, realizar operaciones para conocer el universo

Dar solución a una desigualdad

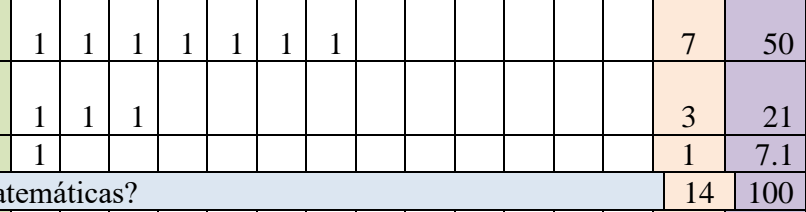

Posibilitar la adquisición de conocimiento ¿Qué es enseñar matemáticas?

matemático, mediante herramientas y métodos, para resolver problemas

Construir actividades para que los estudiantes

identifiquen patrones, relaciones y emitan conjeturas

Dar leyes, hipótesis, axiomas

Motivar a los estudiantes, despertar su interés, por conocer, comprender y aplicar los objetos matemáticos

1

\begin{tabular}{l|l|}
\hline 1 & 7.1 \\
\hline
\end{tabular}

Guiar al estudiante a ver el mundo desde otra perspectiva

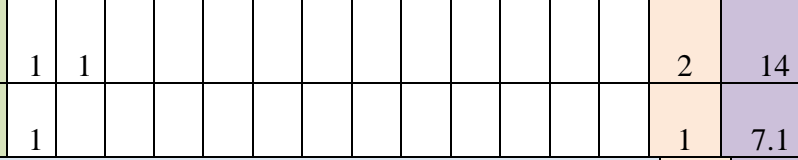
¿Por qué aprender matemáticas?

Las situaciones cotidianas y profesionales involucran el uso de herramientas matemáticas Resolver problemas

Comprender el entorno y mejorarlo

Desarrollar habilidades (razonamiento, generalización) y conocimiento

\begin{tabular}{|l|l|l|l|l|l|}
\hline & 1 & 1 & 1 & 1 & \\
1 & 1 & 1 & & & \\
1 & 1 & 1 & 1 & & \\
& & & & & \\
1 & 1 & 1 & 1 & &
\end{tabular}

$\mid$

\begin{tabular}{|l|l|l|}
\hline & & \\
\hline & & \\
\hline & & \\
\hline & & \\
\hline
\end{tabular}

\begin{tabular}{|l|l|}
\hline & \\
\hline &
\end{tabular}

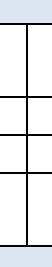

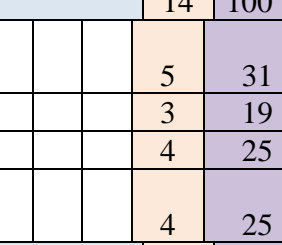

¿Cómo se aprende a resolver desigualdades?

Comparando y aplicando las reglas

Con la explicación conceptual, algebraica, gráfica

\begin{tabular}{c} 
y sus aplicaciones \\
\hline Resolviendo ejercicios
\end{tabular}

\begin{tabular}{c} 
y sus aplicaciones \\
\hline Resolviendo ejercicios
\end{tabular}

\begin{tabular}{lllllllll|l}
1 & 1 & 1 & 1 & 1 & 1 & 1 & 1 & 1 \\
1 & 1 & 1 & & & & & &
\end{tabular}

\begin{tabular}{|l|l|}
\hline 4 & 25 \\
\hline
\end{tabular}

¿Cuáles son los tipos de desigualdades, que más se les complican a los estudiantes y por qué?

$9 \quad 56$

Intersección

Fraccionarias

Valor absoluto

Cuadráticas

Racionales

Dobles

Con números negativos

Con dos variables

Sin solución o con solución infinita

Todas

\begin{tabular}{|l|l|l|l|}
\hline 1 & & & \\
1 & & & \\
1 & 1 & & \\
1 & 1 & 1 & \\
1 & 1 & 1 & \\
1 & 1 & & \\
1 & & & \\
1 & & & \\
1 & & & \\
1 & & &
\end{tabular}

\begin{tabular}{|l|r|}
\hline 16 & 100 \\
\hline
\end{tabular}

\begin{tabular}{|l|l|l|l|l|l|l|l|l|l|l|}
\hline & & & & & & & & & 1 & 6.3 \\
\hline
\end{tabular}

¿Consideras que la herramienta del MOOC ayuda conel aprendizaje del tema de desigualdades?

Accesibilidad permanente

Refuerza el aprendizaje

\begin{tabular}{|r|r|r|r|r|r|r|r|r|r|r|r|r|r|r|}
\hline 1 & 1 & 1 & 1 & 1 & 1 & 1 & 1 & 1 & 1 & 1 & 1 & 1 & 13 & 81 \\
\hline 1 & & & & & & & & & & & & & 1 & 6.3 \\
\hline 1 & 1 & & & & & & & & & & & & 2 & 13 \\
\hline
\end{tabular}

¿Cuántas veces crees que se deba trabajar con el MOOC para atender el tema de desigualdades? Un par

Las veces que sean necesarias Tres

\begin{tabular}{|l|l|l|l|l|l|}
\hline & 1 & 1 & 1 & 1 & \\
\hline 1 & 1 & 1 & 1 & & \\
\hline 1 & & & & &
\end{tabular}


Depende del estudiante

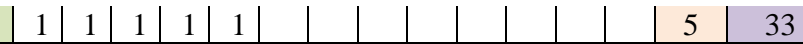
¿Consideras qué los estudiantes preferirían estudiar el tema en el MOOC o con el profesor de forma presencial? MOOC ( como refuerzo o ayuda) Profesor Ambos \begin{tabular}{|l|l|l|}
1 & 1 & \\
1 & 1 & \\
1 & 1 &
\end{tabular} 15 100

(2) ¿Los instrumentos de evaluación del MOOC fueron suficientes y claros?

$17 \quad 100$
sí

No, aumentar y diversificar Depende de la profundidad del tema ¿Cuáles son tus sugerencias para mejorar el MOOC?

\begin{tabular}{|l|l|}
\hline 5 & 29 \\
\hline 8 & 47 \\
\hline 4 & 24 \\
\hline
\end{tabular}

(2)
Actualizar y enriquecer con aplicaciones
Enfocado a estudiantes de preparatoria Escribir introducciones, reglas y ejemplos

\begin{tabular}{|l|l|l|l|l|l|l|l|l|l|l|l|l|l|l|}
1 & 1 & 1 & 1 & 1 & 1 & 1 & 1 & & & & & & 8 & 73 \\
\hline
\end{tabular}

\section{Más ejercicios en cada tema}

Más explícitos en los procedimientos y explicaciones

\section{Emplear colores}

Agregar un apartado de sugerencias y/o comentarios

Incluir videos con estudiantes

Mejorar la calidad de audio y video

\begin{tabular}{|c|c|c|c|c|c|c|c|c|c|c|c|c|c|}
\hline 1 & 1 & & & & & & & & & & & & \\
1 & & & & & & & & & & & & & \\
1 & 1 & 1 & 1 & & & & & & & & & & \\
1 & & & & & & & & & & & & & \\
\hline & & & & & & & & & & & & &
\end{tabular}

\begin{tabular}{l|l}
1 & 9.1 \\
\hline
\end{tabular}

\begin{tabular}{l|r}
\hline 1 & 4.3 \\
\hline
\end{tabular}

\begin{tabular}{|c|}
\hline Emplear colores \\
\hline $\begin{array}{c}\text { Agregar un apartado de sugerencias y/o } \\
\text { comentarios }\end{array}$ \\
\hline Incluir videos con estudiantes \\
\hline Mejorar la calidad de audio y video \\
\hline
\end{tabular}

\begin{tabular}{|rrrrrrrrrrrrrrrr|r|r|r|}
1 & 1 & 1 & 1 & 1 & 1 & 1 & 1 & & & & & & 8 & 35 \\
\hline 1 & 1 & & & & & & & & & & & & 2 & 8.7 \\
\hline 1 & & & & & & & & & & & & & & \\
1 & & & & & & & & & & & & & 1 & 4.3 \\
\hline 1 & 1 & 1 & & & & & & & & & & & 3 & 13 \\
\hline
\end{tabular}

